\title{
Landau type theorem for Orlicz spaces
}

\author{
Lech Maligranda ${ }^{1, *}$ and Witold Wnuk ${ }^{2}$ \\ ${ }^{1}$ Departamento de Matemáticas, IVIC, Apartado 21827, Caracas 1020-A, Venezuela \\ ${ }^{2}$ Institute of Mathematics, Polish Academy of Sciences, Mielżyńskiego 27/29, 61-725 Poznan, \\ Poland
}

Received June 14, 1990; in final form February 25, 1991

\section{Introduction}

In 1907 Edmund Landau [9] proved the reverse Hölder inequality, i.e., if $\left(x_{n} y_{n}\right)_{n=1}^{\infty} \in l^{1}$ for all sequences $\left(x_{n}\right) \in l^{p}(1<p<\infty)$, then $\left(y_{n}\right) \in l^{q}$, where $q$ is the conjugate exponent of $p$. In his original proof he used the following Dini theorem: if $a_{n} \geqq 0$ and $\sum_{1}^{\infty} a_{n}=\infty$ then $\sum_{1}^{\infty}\left(a_{n} / s_{n}\right)=\infty$ and $\sum_{1}^{\infty}\left(a_{n} / s_{n}^{1+\varepsilon}\right)<\infty$ for any $\varepsilon>0$, where $s_{n}$ is the $n$-th partial sum. Now, if there were $\sum_{1}^{\infty}\left|y_{n}\right|^{q}=\infty$, then by the Dini theorem with $x_{n}=\left|y_{n}\right|^{q-1} / \sum_{k=1}^{n}\left|y_{k}\right|^{q}$ we would have $\left(x_{n} y_{n}\right) \notin l^{1}$ but
$\left(x_{n}\right) \in l^{p}$.

Today everybody is able to prove the above result at once using the uniform boundedness principle or the form of linear continuous functionals on $l^{p}$. Landau's theorem belongs to the group of representation theorems of the Köthe dual $E^{x}$ (=associate space in the other terminology) of a Banach function space $E$. The case $E=L^{M}(\mu)$ (i.e., $E$ is an Orlicz space) was thoroughly investigated and it is well-known that $E^{x}=L^{M^{*}}(\mu)$ (see for example [14]), where $M^{*}$ denotes the conjugate function of $M$ (=complementary function in the sense of Young). This fact was originally proved (for convex functions $M$ ) by Birnbaum and Orlicz [3]. The purpose of the paper is to present a direct short and elementary proof of Birnbaum's and Orlicz's result and extend it to Orlicz spaces over atomless or counting measures and generated by finite-valued (not necessarily convex) functions. At the end of the paper one can find an example of an Orlicz space $L^{M}(\mu)$ over purely atomic measure $\mu$ and generated by non-convex function $M$ such that its Köthe dual is not isomorphic to any space of the form $L^{M^{*}}(v)$.

We refer readers interested in Orlicz spaces to $[8,11,14]$.

Let $(\mathrm{S}, \Sigma, \mu)$ be a $\sigma$-finite measure space. Moreover, assume that $M:[0, \infty)$ $\rightarrow[0, \infty]$ is a non-decreasing function and $M(0)=0$.

* Current address: Department of Mathematics, Luleå University of Technology, S-95187 Luleå, Sweden 
If $L^{0}=L^{0}(S, \Sigma, \mu)$ denotes the space of $\Sigma$-measurable functions (with the usual identification of functions equal $\mu$-almost everywhere) then

$$
L^{M}(\mu)=\left\{f \in L^{0}: m_{M}(r f)=\int_{S} M(r|f(s)|) d \mu<\infty \text { for some } r>0\right\}
$$

is a linear subspace of the space $L^{0}$ (called the Orlicz space) and the functional

$$
\|f\|=\inf \left\{a>0: m_{M}(f / a) \leqq a\right\}
$$

is a monotone group semi-norm on the space $L^{M}(\mu)$, i.e., $\|0\|=0,\|f+g\| \leqq\|f\|$ $+\mid g \|$ and $|f| \leqq|g| \mu$-almost everywhere implies $f\|\leqq\| g \|$. It is clear that the topology determined by $\|\cdot\|$ is Hausdorff iff $M$ is not identically zero. Moreover, $\|\cdot\|$ is an $F$-norm on $L^{M}(\mu)$ iff $M$ is continuous at zero or $M(r)=\infty$ for $r>0$. In papers devoted to Orlicz spaces authors usually assume that $M$ is left continuous, continuous at zero, non-decreasing and $M(0)=0$. If $M$ is convex continuous at zero and $M \neq 0$, then the topology determined by $\|\cdot\|$ is equivalent to norm topology generated by $\|f\|=\inf \left\{a>0: m_{M}(f / a) \leqq 1\right\}$. The $F$-norm $\|\cdot\|$ is always complete.

We will often write $L^{M}$ instead of $L^{M}(\mu)$ and $l^{M}$ when $\mu$ is the counting measure on subsets of natural numbers, i.e., $l^{M}$ is an Orlicz sequence space.

Let us distinguish the ideal $L_{A}^{M} \subset L^{M}$ consisting of elements with order continuous norm, i.e., $L_{A}^{M}=\left\{f \in L^{M}:|f| \geqq f_{n} \downarrow 0\right.$ implies $\left.\left\|f_{n}\right\| \rightarrow 0\right\}$. If $\left(S_{n}\right)$ is a sequence of atoms of finite measure $\mu$ such that $\mu$ restricted to measurable subsets of $S \backslash \bigcup_{n=1}^{\infty} S_{n}$ is atomless, then

$$
\begin{aligned}
L_{A}^{M}= & \left\{f \in L^{M}: \operatorname{supp} f \in S \backslash \bigcup_{n}^{\infty} S_{n} \text { and } m_{M}(r f)<\infty \text { for all } r \text { 's }\right\} \\
& \oplus \operatorname{span}\left\{1_{S_{n}}: n \in N\right\},
\end{aligned}
$$

where $1_{S_{n}}$ is the characteristic function of the set $S_{n}$.

For a function $M$ we define the conjugate function $M^{*}$ of $M$ by

$$
M^{*}(r)=\sup \{s r-M(s): s \geqq 0\},
$$

(we put $x-\infty=-\infty$ for every real number $x$ ). The function $M^{*}$ is always convex and left continuous. The notation $M^{* *}$ means the function $M^{* *}=\left(M^{*}\right)^{*}$. After simple computations we obtain a useful inequality

$$
M^{* *}\left(M^{*}(u) / u\right) \leqq M^{*}(u)
$$

(we understand $M^{* *}(\infty)$ as $\infty$ ). We always have $M^{* *} \leqq M$ and $M^{*}=M^{* * *}$.

Since we also consider Orlicz spaces $L^{M}(\mu)$ which are not Banach spaces let us quote the exact definition of the Köthe dual $\left(L^{M}(\mu)\right)^{x}=\left(L^{M}\right)^{x}$ of $L^{M}(\mu)$ :

$$
\left(L^{M}\right)^{x}=\left\{g \in L^{0}(S, \Sigma, \mu): f \cdot g \in L^{1}(\mu) \text { for all } f \in L^{M}(\mu)\right\} .
$$

The space $\left(L^{M}\right)^{x}$ is always a Banach space with respect to the norm

$$
\|g\|=\sup \left\{\|f \cdot g\|_{L^{1}}: m_{M}(f) \leqq 1\right\} .
$$




\section{Main results}

Theorem 1 If a function $M \neq 0$ is convex and left continuous on $(0, \infty)$ then $\left(L^{M}\right)^{x}=L^{M^{*}}$.

Proof. It is sufficient to prove $\left(L^{M}\right)^{x} \subset L^{M^{*}}$ because the reverse inclusion is a consequence of the inequality $r s \leqq M(r)+M^{*}(s)$.

Suppose $g \in L^{0}$ is such that $\bar{f} \cdot g \in L^{1}$ for all $f \in L^{M}$. Therefore $g$ determines a continuous linear functional $G$ on $L^{M}$ by the equality $G(f)=\int_{S} f \cdot g d \mu$. Put
$c=\|G\|$.

Let us consider two cases:

Define

1. $M^{*}(r)<\infty$ for all $r$ 's.

$$
g_{0}(s)= \begin{cases}\frac{c+1}{|g(s)|} M^{*}\left(\frac{|g(s)|}{c+1}\right) & \text { for } g(s) \neq 0 \\ 0 & \text { for } g(s)=0\end{cases}
$$

There is no loss of generality to assume $M(u)$ is finite at some point $u>0$ (if $M(r)=\infty$ for $r>0$ then $\left(L^{M}\right)^{x} \subset L M^{*}=L^{0}$ and we are done). It is clear that the support of $L^{M}$ equals $S$ and so we can choose a sequence $\left(S_{n}\right)$ of measurable sets with the following properties: $S_{n} \subset S_{n+1}, S=\bigcup_{n=1}^{\infty} S_{n}, 0<\mu\left(S_{n}\right)<\infty$ and $g_{0} \cdot 1_{S_{n}} \in L^{M}$ (see $[7$, p. 136, Corollary 1]).

Since $M$ is convex and left continuous we have $M=M^{* *}$ in virtue of the Fenchel-Moreau theorem ([2, p. 86, Theorem 1.4] or [6, p. 186, Theorem 1]) and therefore using inequality $(*)$ we obtain

$$
m_{M}\left(g_{0} 1_{S_{n}}\right) \leqq m_{M^{*}}\left(\frac{|g(\cdot)|}{c+1}\right)=\int_{S_{n}} g_{0}(s)|g(s)|(c+1)^{-1} d \mu<\infty
$$

because $g_{0} 1_{S_{n}} \in L^{M}$. We claim $m_{M}\left(g_{0}\right)<\infty$. If $m_{M}\left(g_{0}\right)$ were infinite then $b=$ $m_{M}\left(g_{0} 1_{S_{n}}\right)>2$ for some $n$ in virtue of $m_{M}\left(g_{0} 1_{S_{n}}\right) \uparrow m_{M}\left(g_{0}\right)$. Moreover, the convexity of $M$ implies $b^{-1}\left\|g_{0} 1_{S_{n}} \mid\right\| \leqq 1$ and thus

$$
\begin{aligned}
b & =m_{M}\left(g_{0} 1_{S_{n}}\right) \leqq \int_{S_{n}} g_{0}(s)|g(s)|(c+1)^{-1} d \mu \\
& =b(c+1)^{-1} \int_{S_{n}}\left(b^{-1} g_{0}(s)\right)|g(s)| d \mu \leqq b c(c+1)^{-1}<b
\end{aligned}
$$

and we have got a contradiction. Therefore, it has to be $m_{M}\left(g_{0}\right)<\infty$ which implies $g \in L^{M^{*}}$.

2. There exists $r_{0}>0$ such that $M^{*}(r)<\infty$ for $r<r_{0}$ and $M^{*}(r)=\infty$ for $r>r_{0}$. We claim $g \in L^{\infty}$. Indeed, let $r>r_{0}$ be fixed. We have

$$
\infty=\sup \{s r-M(s): s \geqq 0\}=\sup \{s r-M(s): s>n\}
$$

and so there is a sequence $\left(s_{n}\right)$ increasing to infinity with $r s_{n}-M\left(s_{n}\right)>0$, i.e., $M\left(s_{n}\right) / s_{n}<r$. For a number $s>0$ choose an index $n_{0}$ such that $s<s_{n_{0}}$. Since 
the function $M(x) / x$ is non-decreasing we obtain $M(s) / s \leqq M\left(s_{n_{0}}\right) / s_{n_{0}}<r$. Finally $M(s) \leqq r s$. Hence $L^{1} \subset L^{M}$ and this inclusion implies $g \in L^{\infty}$. Putting

$$
g_{0}(s)= \begin{cases}\frac{d}{|g(s)|} M^{*}\left(\frac{|g(s)|}{d}\right) & \text { for } g(s) \neq 0 \\ 0 & \text { for } g(s)=0\end{cases}
$$

where $d=\max \left(c+1,\left(\|\mathrm{~g}\|_{\infty}+1\right) r^{-1}\right)$, and repeating the arguments used in part 1 we will also obtain $g \in L^{M^{*}}$.

Remark 1 Let us note that the method used in the first part of the proof is similar to that in [10, Theorem 4].

Theorem 2 If $M \neq 0$ is finite-valued, left continuous, continuous at zero, nondecreasing and $\mu$ is atomless, then $\left(L^{M}\right)^{x}=L^{M^{*}}$.

Proof. We have only to prove $\left(L^{M}\right)^{x} \subset L^{M^{*}}$. The inequality $M^{* *} \leqq M$ implies $L^{M} \subset L^{M^{* *}}$. Let us consider two possibilities:

1. There exists a positive number $r$ with $M^{* *}(r)>0$.

Let $g \in\left(L^{M}\right)^{x}$ and let $G$ be the functional determined by $|g|$, i.e., $G(f)=\int_{S} f|g| d \mu$.

This functional restricted to $L_{A}^{M}$ remains continuous with respect to the topology $\tau^{* *}$ induced from $L_{A}^{M^{* *}}\left(\left[4\right.\right.$, Theorem 2]). The support of $L_{A}^{M}$ equals $S$, and so every positive function $f \in L^{M^{* *}}$ is the supremum of some increasing sequence $\left(f_{n}\right)$ of positive functions from $L_{A}^{M}$. Since $\left(f_{n}\right)$ is $\tau^{* *}$-bounded and $G$ is $\tau^{* *}$-continuous we have $\int_{S} f|g| d \mu=\sup _{n} \int_{S} f_{n}|g| d \mu<\infty$, i.e., $f \cdot g \in L^{1}$ for all $f \in L^{M^{* *}}$. Therefore $g \in L^{M^{* * *}}$ in virtue of Theorem 1 and we are done because $L^{M^{* * *}}=L^{M^{*}}$.

2. $M^{* *} \equiv 0$.

In this situation $M^{*}(r)=\infty$ for $r>0$, i.e., $L^{M^{*}}=\{0\}$. Moreover, $\lim \inf M(r) / r=0$.

The last equality gives $\left(L^{M}\right)^{x}=\{0\}([4$, Corollary 1$])$. Finally $\left(L^{M}\right)^{x}=L^{M^{*}}$.

Remark 2 The assumption that $\mu$ is atomless is essential. If $M(r)=r^{p}(0<p<1)$ then $M^{*}(r)=\infty$ for $r>0$ and the Orlicz sequence space $l^{M^{*}}$ is trivial, but $\left(l^{p}\right)^{x}=l^{\infty}$.

Now we will pay attention to the case of Orlicz sequence spaces $l^{M}$. Let us recall that functions $M$ and $N$ are equivalent at zero if there exist positive constants $a, b, c, d, x$ such that

$$
a M(b u) \leqq N(u) \leqq c M(d u) \quad \text { for } u \in[0, x]
$$

Since properties of a space $l^{M}$ are determined by the behaviour of $M$ in a neighborhood of zero we can assume that $M$ is finite-valued.

Theorem 3 If $M$ has all properties listed in Theorem 2, then $\left(l^{M}\right)^{x}=l^{M *}$, where $M_{\propto}(u)=M(u)$ for $0 \leqq u \leqq 1$ and $M_{\infty}(u)=\infty$ for $u>1$.

(The notation $M_{\infty}^{*}$ means the conjugate function of $M_{\infty}-$ not the function $\left.\left(M^{*}\right)_{\infty}\right)$.

Proof. Let us consider two cases:

1. $M(u)=0$ iff $u=0$. 
Denote by $\hat{M}$ the convex minorant of the function $M$ in the interval $[0,1]$, i.e., $\widehat{M}(t) \leqq M(t)$ for all $t \in[0,1]$ and if $N$ is a convex function on $[0,1]$ and it satisfies $N(t) \leqq M(t)$ for $t \in[0,1]$ then $N(t) \leqq \hat{M}(t)$ for all $t \in[0,1]$. Putting $\hat{M}(u)=\infty$ for $u>1$ we obtain that $\hat{M}$ is convex on the half line $[0, \infty)$ and $\hat{M} \leqq M_{\infty}$. Thus $\hat{M} \leqq M_{\infty}^{* *}$ and $l^{M}=l M_{\infty} \subset l^{M * *} \subset l^{\hat{M}}$.

Let $\left(y_{n}\right)$ be a sequence of positive numbers belonging to $\left(l^{M}\right)^{x}$. The functional $G$ defined on $l_{A}^{M}$ by the formula $G\left(\left(x_{n}\right)\right)=\sum_{1}^{\infty} x_{n} y_{n}$ is continuous with respect to the topology induced from $l_{A}^{\hat{A}}$ (see [5, Theorem 5.1]), and so it will remain continuous with respect to the topology induced from $l_{A}^{M^{* *}}$ because this topology is stronger.

If $0<x=(x(k)) \in l^{M * *}$, then there exists a sequence $\left(x_{n}\right)$ of elements from $l_{A}^{M}$ such that $0 \leqq x_{n} \uparrow x$. Since the sequence $\left(x_{n}\right)$ is bounded in the topology induced from $l_{A}^{M_{\infty}^{* *}}$, then $c=\sup G\left(x_{n}\right)<\infty$. Using the famous Fatou lemma we will obtain $c=\sum_{1}^{\infty} x(k) y(k)$. Therefore $\left(y_{n}\right) \in\left(l^{M_{\infty}^{* *}}\right)^{x}=l^{M_{\infty}^{* * *}}=l^{M_{\infty}^{*}}$ (the first equality follows from Theorem 1). In other words $\left(l^{M}\right)^{x} \subset l^{M_{x}^{*}}$.

On the other hand the inclusion $l^{M_{\infty}} \subset l^{M_{\infty}^{* *}}$ gives $l^{M_{\infty}^{*}}=\left(l^{M_{\infty}^{* *}}\right)^{x} \subset\left(l^{M_{\infty}}\right)^{x} \subset\left(l^{M}\right)^{x}$.

Finally $\left(l^{M}\right)^{x}=l^{M_{\infty}^{*}}$.

2. $M([0, t])=\{0\}$ for some $t>0$.

It is obvious that $l^{M}=l^{\infty}$, and so $\left(l^{M}\right)^{x}=l^{1}$. The proof will be finished if we show $l^{M_{\infty}^{*}}=l^{1}$.

Suppose first $t \geqq 1$. Under this assumption we have $M_{\infty}^{*}(u)=\sup \{u v$ $-M(v): 0 \leqq v \leqq 1\}=u$, i.e., $l^{M_{\infty}^{*}}=l^{1}$.

If $t<1$ then $M_{\infty}^{*}(u) \geqq \sup \{u v-M(v): 0 \leqq v \leqq t\}=t u$. The convexity of the function $M_{\infty}^{*}$ implies $M_{\infty}^{*}(u) \leqq M_{\infty}^{*}(1) u \leqq u$ for $u \in[0,1]$. Therefore $M_{\infty}^{*}$ is equivalent at zero to the function $N(u)=u$ and thus $l^{M_{\infty}^{* *}}=l^{1}$ and we are done.

Remark 3 If $M(1)>0$ and $\liminf _{u \rightarrow \infty} M(u) / u>0$, then $M_{\infty}^{*}(u)=M^{*}(u)$ for $u$ belonging to some neighborhood of zero and therefore $l^{M_{\infty}^{*}}=l^{M^{*}}$,

Indeed, supposing $\lim \inf M(u) / u>0$ we find numbers $a>0, v_{0}>1$ such that $M(v) / v>a$ for all $v \geqq v_{0}$. Taking $v \in\left(1, v_{0}\right)$ we have $M(v) / v \geqq M(1) / v_{0}>0$, and so $\left.\inf \{M(v) / v: v>1\} \geqq \min \left(a, M(1) / v_{0}\right)\right)$. The following inequalities are valid for $u \in\left[0, \min \left(a, M(1) / v_{0}\right)\right)$ :

$$
\begin{aligned}
M^{*}(u) & =\sup \{u v-M(v): v \geqq 0\}=\sup \{u v-M(v): 0 \leqq v \leqq 1\} \\
& =\sup \left\{u v-M_{\infty}(v): 0 \leqq v \leqq 1\right\}=\sup \left\{u v-M_{\infty}(v): v \geqq 0\right\}=M_{\infty}^{*}(u) .
\end{aligned}
$$

The assumption $M(1)>0$ is essential: if $N(u)=0$ for $0 \leqq u \leqq 2$ and $N(u)=$ $u^{2}-4$ for $u>2$, then $N^{*}(u) \geqq \sup \{u v-N(v): 0 \leqq v \leqq 2\}=2 u$ and $N_{\infty}^{*}(u)=u$, and so the functions $N^{*}$ and $N_{\infty}^{*}$ are different, althogh they are equivalent at zero.

Remark 4 If $\liminf _{u \rightarrow \infty} M(u) / u=0$ then $M^{*}(u)=\infty$ for $u>0$.

We can find a sequence $\left(u_{n}\right)$ tending to infinity such that $\left(M\left(u_{n}\right) / u_{n}\right)$ converges to zero. Therefore, fixing $r>0$ and taking sufficiently large $n$ we will obtain $M^{*}(r) \geqq u_{n}\left(r-\left(M\left(u_{n}\right) / u_{n}\right)\right) \geqq 2^{-1} r u_{n}$, i.e., $M^{*}(r)=\infty$. 
Remark 5 If $\lim \inf M(u) / u=0$ (in particular, if $M$ is concave) then $l^{M_{\infty}^{*}}=l^{\infty}$. Indeed, the inequality $\lim \inf M(u) / u>0$ guarantees the existence of positive numbers $c$ and $u_{0}$ such that $M(u) / u>c$ for $0<u \leqq u_{0}$. Denoting $c_{0}=\inf \{M(v)$ : $\left.u_{0} \leqq v \leqq 1\right\}$ we obtain $M_{\infty}^{*}(u)=0$ for $u<\min \left(c, c_{0}\right)$. Moreover $M_{\infty}^{*}$ is not identically equal to zero. If there were $M_{\infty}^{*}(u)=0$ for $u>0$, then we would have $u v \leqq M(v)$ for all $u>0$ and all $v \in[0,1]$. In particular, $u \leqq M(1)$ for all $u$ and we have got a contradiction because we consider finite-valued functions only. Finally, the facts that $M_{\infty}^{*}$ vanish in some neighborhood of zero but $M_{\infty}^{*}$ is not identically zero imply $l^{M_{\infty}^{*}}=l^{\infty}$.

Remark 6 Theorem 3 (under slightly stronger assumptions about $M$ ) was also proved (as a corollary of more general results) by $M$. Nowak (see [12, Theorem 3.3]) but his proof is more complicated.

Let us note assumptions about measures in Theorems 2 and 3 are important. There exists an Orlicz space $L^{M}(\mu)$ over a purely atomic measure $\mu$ with $M$ non-convex such that the Köthe dual $\left(L^{M}(\mu)\right)^{x}$ is not isomorphic to the space $L^{M *}(v)$ for any measure $v$.

Theorem 4 Let $M$ have all properties listed in Theorem 2 and suppose $M$ satisfies the following conditions: $M(\boldsymbol{u})=0$ iff $\boldsymbol{u}=0, \limsup _{\boldsymbol{u} \rightarrow \infty} M(2 u) / \boldsymbol{u}=\infty$ and $M^{* *}(\boldsymbol{u})=\boldsymbol{u}$.

There exists a purely atomic measure $\mu$ such that $\left(L^{M}(\mu)\right)^{x}$ is not isomorphic to the space $L^{M^{*}}(v)$ for any measure $v$.

Proof. Using [13, Theorem 1] we can choose a sequence $\left(a_{n}\right)$ of positive numbers such that defining the measure $\mu$ on subsets of natural numbers by the formula $\mu(A)=\sum_{n \in A} a_{n}$ we will have that $L^{M}(\mu)$ is order isomorphic to $l^{\infty}$. Therefore $\left(L^{M}(\mu)\right)^{x}$ and $l^{1}$ are order isomorphic. If the spaces $\left(L^{M}(\mu)\right)^{x}$ and $L^{M^{*}}(v)$ were isomorphic for some measure $v$, then $L M^{*}(v)$ would be order isomorphic to $l^{1}$ on account of $\left[1\right.$, Theorem 5]. Thus by Theorem 1 the space $L^{M^{* *}}(v)=\left(L^{M^{*}}(v)\right)^{x}$ would be order isomorphic to $l^{\infty}$, but this is impossible because $M^{* *}(\boldsymbol{u})=u$ implies $L^{M^{* *}}(v)=L^{1}(v)$.

Example of the function $M$ satisfying the assumptions of Theorem 4 . Let

$$
\begin{aligned}
& a_{n}= \begin{cases}2^{n(n-1) / 2} & \text { for odd } n \text { 's } \\
2^{(n-2)(n-1) / 2}+1 & \text { for even } n \text { 's }\end{cases} \\
& b_{n}= \begin{cases}2^{n(n-1) / 2}+1 & \text { for odd } n \text { 's } \\
2^{(n+1) / 2} & \text { for even } n \text { 's. }\end{cases}
\end{aligned}
$$

Define a function $M$ by the formula

$$
M(u)= \begin{cases}u & \text { if } 0 \leqq u \leqq 1 \\ 2^{n(n+1) / 2} & \text { if } a_{n}<u \leqq b_{n}\end{cases}
$$

It is easy to see that $M$ is left continuous, continuous at zero, non-decreasing and $M$ vanish only at zero. Putting $u_{n}=b_{2 n-1}$ we get $2 u_{n} \in\left(a_{2 n} b_{2 n}\right]$ and

$$
M\left(2 u_{n}\right) / M\left(u_{n}\right)=2^{2 n} \rightarrow \infty \text {, i.e., } \limsup _{u \rightarrow \infty} M(2 u) / u=\infty \text {. }
$$


Suppose that $N$ is a convex function defined on $[0, \infty)$ satisfying the inequality $N(u) \leqq M(u)$ for all $u$ 's. If we show that $N(u) \leqq u$, then the function $\tilde{M}(u)=u$ will be the convex minorant of $M$ on account of the inequality $u \leqq M(u)$. Therefore $M^{* *}(u)=u$ because $M^{* *}$ is the greatest convex function on $[0, \infty)$ not exceeding $M$.

Assume first $u \geqq 2$. Since $u \in\left[b_{2 k-1}, b_{2 k+1}\right]$ for some $k$ then $u=c b_{2 k-1}$ $(1-c) b_{2 k+1}$ for some $c \in[0,1]$. Thus

$$
\begin{aligned}
& N(u) \leqq c N\left(b_{2 k-1}\right)+(1-c) N\left(b_{2 k+1}\right) \leqq c M\left(b_{2 k-1}\right)+(1-c) M\left(b_{2 k+1}\right) \\
& =c b_{2 k-1}+(1-c) b_{2 k+1}=u
\end{aligned}
$$

If $1<u \leqq 2$ then repeating the above arguments with $1=b_{2 k-1}$ and $2=b_{2 k+1}$ we obtain $N(u) \leqq u$. Finally, we have $N(u) \leqq M(u)=u$ for $0 \leqq u \leqq 1$. Thus $N(u) \leqq u$ on the half line and we are done.

Acknowledgement. The authors are grateful to Professor Marek Nawrocki who has explained the idea of construction of the function $M$ in the above example.

\section{References}

1. Abramovič, Ju.A., Wojtaszczyk, P.: The uniqueness of the order in spaces $L_{p}[0,1]$ and $l_{p}$ (in Russian). Mat. Zametki 18, 313-325 (1975)

2. Barbu, V., Precupanu, Th.: Convexity and Optimization in Banach Spaces. Bucharest: Sijthoff and Noordhoff 1978

3. Birnbaum, Z.W., Orlicz, W.: Über die Verallgemeinerung des Begriffes der zueinander konjugierten Potenzen. Stud. Math. 3, 1-67 (1931); reprinted in: Orlicz, W.: Collected Papers, pp. 133-199. Warsaw: Polish Scientific Publishers 1988

4. Drewnowski, L.: Compact operators on Musielak-Orlicz spaces. Ann. Soc. Math. Pol., Ser. I, Commentat. Math. 27, 225-232 (1988)

5. Drewnowski, L., Nawrocki, M.: On the Mackey topology of Orlicz sequence spaces. Arch. Math. 39, 59 $68(1982)$

6. Ioffe, A.D., Tikhomirov, V.M.: Theory of Extremal Problems (in Russian). Moscow: Nauka 1974

7. Kantorovič, L.V., Akilov, G.P.: Functional Analysis (in Russian). Moscow: Nauka 1984

8. Krasnosel'skii, M.A., Rutickii, Ya.B.: Convex Functions and Orlicz Spaces. Groningen: Noordhoff 1961

9. Landau, E.: Über einen Konvergenzsatz. Nachr. Königl. Ges. Wiss. Göttingen, Nr.8, 25-27 (1907)

10. Maligranda, L., Persson, L.E.: Generalized duality of some Banach function spaces. Indagationes Math. 51, 323-338 (1989)

11. Musielak, J.: Orlicz Spaces and Modular Spaces (Lect. Notes Math., vol. 1034). Berlin Heidelberg New York: Springer 1983

12. Nowak, M.: The Köthe dual of Orlicz sequence spaces without local convexity. Math. Japonica 34, 619-627 (1989)

13. Wnuk, W.: Orlicz spaces which are Riesz isomorphic to $l^{\infty}$. Rocky Mt. J. Math. 18, 185-193 (1988)

14. Zaanen, A.C.: Riesz Spaces II. Amsterdam New York Oxford: North-Holland 1983 